\title{
THE EMBEDDED TRIANGLES ALGORITHM FOR DISTRIBUTED ESTIMATION IN SENSOR NETWORKS
}

\author{
V. Delouille, R. Neelamani, V. Chandrasekaran, and R. G. Baraniuk \\ Department of Electrical and Computer Engineering \\ Rice University
}

\begin{abstract}
We propose a new iterative distributed estimation algorithm for Gaussian hidden Markov graphical models with loops. We decompose a loopy graph into a number of linked embedded triangles and then apply a parallel block-Jacobi iteration comprising local linear minimum mean-square-error estimation on each triangle (involving a simple $3 \times 3$ matrix inverse computation) followed by an information exchange between neighboring nodes and triangles. A simulation study demonstrates that the algorithm converges extremely rapidly, outperforming a number of existing algorithms. Embedded triangles are simple, local, scalable, fault-tolerant, and energy-efficient, and thus ideally suited for wireless sensor networks.
\end{abstract}

\section{INTRODUCTION}

Sensor technologies, signal processing, and wireless communication have matured to the point where large networks of sensor nodes can now be easily deployed in a wide variety of environments, making them very attractive for large-scale applications like environmental monitoring, security surveillance, and disaster relief [1]. Often battery-powered, sensor nodes are capable of sensing, computing, and communicating information.

In the setting considered here, each sensor node makes noisy scalar measurements of its physical environment, such as temperature, wind speed, or concentration of some substance. A core sensor network problem involves producing accurate estimates of the true values being sensed from the noisy measurements.

A naive estimation approach would first transmit all measurements to a central location and then apply classical estimation theory, for example lithear minimum mean-square-error (LMMSE) Wiener estimation for Gaussian measurements. For $N$ sensor nodes, this involves computing an $N \times N$ matrix inverse to solve the associated normal equations. Unfortunately, since communication consumes significant energy [2], the naive centralized approach is extremely wasteful. Thus, there is a great need for distributed estimation algorithms that replace global communication and centralized computation by local communication and computation, effectively distributing the $N \times N$ matrix inverse computation across the network.

Graphical models provide a natural setting for developing distributed estimation algorithms. Here, the correlations between sensor measurements are encoded in a graph structure. For example, a reasonable model assumes that inter-sensor correlations decay monotonically with distance and simply approximates a net-

This work was supported by grants from NSF, ONR, AFOSR, DARPA, and the Texas Instruments Leadership University Program. Email: $\{$ vero,neelsh,venkatc,richb $\} @$ rice.edu. Web: dsp.rice.edu. work's correlation structure in terms of a triangulated graph (see Fig. 1(a)) [3,4].

An important characteristic of the graphs associated with most sensor networks is the presence of loops, which greatly complicate distributed estimation [5,6]. A number of distributed estimation approaches for loopy graphs have been proposed, including belief propagation (BP) [7], loopy BP [5], Gaussian elimination message passing [6], and embedded trees $[8,9]$. However, these algorithms either are complicated, converge to biased values, converge slowly, or are not suitable for wireless sensor networks.

In this paper, we propose a new algorithm for distributed LMMSE estimation that is simple, local, scalable, fault-tolerant, and energy-efficient, and thus ideally suited for wireless sensor networks. The core idea is to decompose a loopy graphical model in terms of a number of linked embedded triangles. The iterative algorithm then comprises local LMMSE estimation on each triangle (a $3 \times 3$ matrix inverse computation) followed by an exchange of this information between neighboring nodes and triangles. The algorithm converges extremely rapidly in terms of both number of iterations and total communication energy expended, outperforming a number of other algorithms in the literature.

This paper is organized as follows. Section 2 introduces a statistical hidden Markov model (HMM) for a sensor network; Section 3 outlines two current distributed techniques for estimation under this model. Section 4 develops the embedded triangles algorithm, while Section 5 illustrates its performance in a simulation experiment. Section 6 reviews the advantages of embedded triangles, and Section 7 concludes.

\section{ESTIMATION IN SENSOR NETWORKS}

\subsection{Graphical models}

Graphical models, including Bayesian networks and Markov random fields, represent statistical dependencies between variables by means of a graph [4]. Let $\mathcal{G}$ be an undirected graph defined by a set of nodes $\mathcal{V}$ and a set of edges $\mathcal{E}$. Each node $s \in \mathcal{V}$ is associated with an unobserved or hidden variable $x_{s}$ as well as with a noisy measurement $y_{\mathrm{s}}$. We assume that, given the set of hidden variables $x:=\left\{x_{s} \mid s \in \mathcal{V}\right\}$, the observations $y:=\left\{y_{s} \mid s \in \mathcal{V}\right\}$ are independent of each other. A missing edge $(i, j)$ between nodes $i$ and $j$ implies conditional independence between the variables $x_{i}$ and $x_{j}$ given all the other hidden variables. That is, if the neighborhood of node $s$ is defined as $\mathcal{N}(s):=\{t \mid(s, t) \in \mathcal{E}\}$, then $p\left(x_{s} \mid x_{\mathcal{V} \backslash s}\right)=p\left(x_{s} \mid x_{\mathcal{N}(s)}\right)$.

In this paper, we focus on the case where $x$ is a jointly Gaussian process; such a model is called a Gaussian hidden Markov model (HMM). Let $x \sim N(0, \Sigma)$ be an unobserved random vector with conditional independence relations described by the graphical model $\mathcal{G}=(\mathcal{V}, \mathcal{E})$. Setting $P=\Sigma^{-1}$, the $(s, t)$-th entry of $P$, denoted $P_{s, t}$, will be nonzero if and only if the edge $(s, t) \in \mathcal{E}[4,9]$. 




(a) Sensor network (Delaunay triangulated)

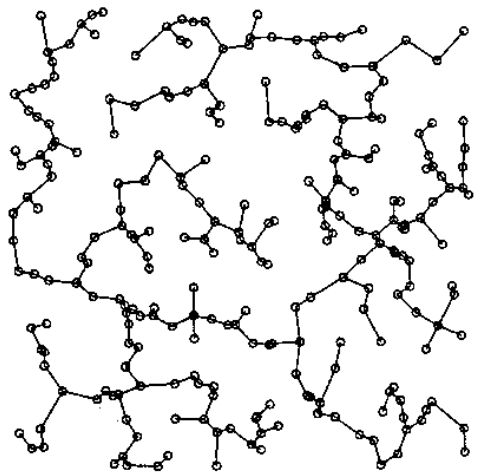

(b) Minimum spanning tree

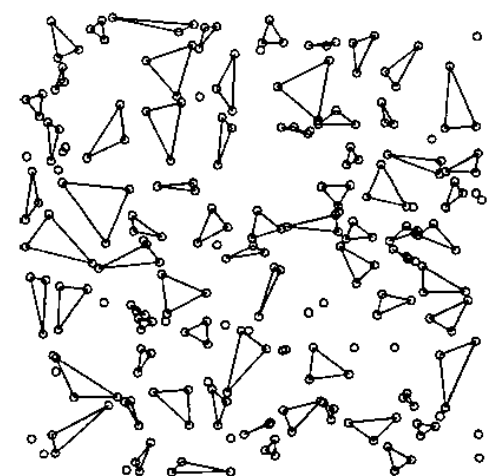

(c) A set of independent embedded triangles

Fig. 1. Example sensor network with 250 nodes.

\subsection{Estimation for HMMs}

Given a vector of noisy observations $y=x+\epsilon, \epsilon \sim N(0, R)$, the minimum mean-square error estimate of $x$ is provided by the posterior conditional distribution $p(x \mid y) \sim N(\widehat{x}, \widehat{\Sigma})$ and computed from the normal equations

$$
V \widehat{x}=R^{-1} y
$$

Here $V:=\Sigma^{-1}+R^{-1}=P+R^{-1}$ and $\widehat{\Sigma}=V^{-1}$. Since $\widehat{x}$ is a linear function of $y$, we use the term LMMSE estimate.

\subsection{Sensor networks and Delaunay triangulation}

When the process $x$ being sensed is Gaussian and varies smoothly across space so that neighboring sensors measure similar values, a Gaussian HMM for the noisy measurements $y$ is natural. To specify the HMM matrix $\Sigma$, note that $P_{s, t}$ is related to the partial correlation $r_{s, t \mid \mathcal{V} \backslash\{s, t\}}$ between $x_{s}$ and $x_{t}$ [4]: $r_{s, t \mid \mathcal{V} \backslash\{s, t\}}=$ $-P_{s, t} / \sqrt{P_{s s} P_{t t}}$. Thus, if the measured field varies smoothly across space, then choosing $r_{s, t \mid \mathcal{V} \backslash\{s, t\}}$ as a decreasing function of the distance between $s$ and $t$ is reasonable [3].

In order to capture the spatial dependencies between the observations taken at neighboring sensors, we propose a graphical model based on the Delaunay triangulation [10] of the sensor locations in space. Figure 1(a) illustrates a sensor network and its Delaunay triangulation. Note that while we focus on Delaunay graphs in this paper, the methods developed below can easily be extended to arbitrary loopy topologies. We will use the terms "sensor" and "node" interchangeably below.

\section{DISTRIBUTED HMM ESTIMATION}

A number of distributed message passing algorithms have been proposed for solving (1), including belief propagation (BP) [7], loopy BP [5], and extended message passing based on Gaussian elimination [6]. We will focus instead on a very attractive class of iterative methods for solving (1) based on a decomposition of $V$.

\subsection{Matrix splitting algorithms}

Let $\bar{y}=R^{-1} y$ denote the normalized observation vector. Equation (1) then becomes

$$
V \widehat{x}=\bar{y} .
$$

If we rewrite $V$ by "splitting" it into $V=J-K$, then solving (2) is equivalent to finding a fixed point of the system $J \widehat{x}=\bar{y}+$
$K \widehat{x}$. Starting from an initial guess $\widehat{x}^{0}$, this suggests we generate a sequence of iterates $\left\{\widehat{x}^{m}\right\}_{m=1}^{\infty}$ according to the recursion

$$
\begin{aligned}
\text { Update: } & \widehat{y}^{m}=\bar{y}+K \widehat{x}^{m-1} \\
\text { Solve: } & \widehat{x}^{m}=J^{-1} \widehat{y}^{m}, \quad m=1,2, \ldots
\end{aligned}
$$

This algorithm converges when the spectral radius of the matrix $J^{-1} K$ is strictly smaller than one: $\widehat{x}^{m} \stackrel{m \rightarrow \infty}{\rightarrow} \widehat{x} \Leftrightarrow \rho\left(J^{-1} K\right)<$ $1[11]$.

For sensor networks, we seek a $(J, K)$ pair for which the Update and Solve steps can be performed by simple computations involving information only from neighboring nodes. We assume that each sensor $s \in \mathcal{G}$ knows its current estimate $\widehat{x}_{s}^{m}$ as well as the partial correlations with its neighbors; that is, the sensor $s$ knows row $s$ of the matrix $V$. Let $\mathcal{N}_{K}(s):=\left\{t \in \mathcal{N}(s) \mid K_{s t} \neq 0\right\}$ be the neighbors of $s$ that correspond to nonzero entries in $K$. To compute the Update (3), each node $s$ needs to receive from its neighbors $\mathcal{N}_{K}(s)$ their current estimates $\widehat{x}_{\mathcal{N}_{K}(s)}^{m-1}$.

\subsection{Jacobi algorithm}

The simplest splitting algorithm is the well-known Jacobi iteration that sets $J$ equal to the diagonal of $V$. Iteration $m$ proceeds as:

Update: Node $s$ sends its current estimate $\widehat{x}_{s}^{m-1}$ to its neighbors $j \in \mathcal{N}(s)$ and receives the current estimates $\widehat{x}_{j}^{m-1}$ from those same neighbors.

Solve: $\widehat{x}_{s}^{m}=V_{s s}^{-1}\left(\bar{y}_{s}-\sum_{t \in \mathcal{N} s} V_{s, t} x_{t}^{m-1}\right) \forall s \in \mathcal{G}$.

The Jacobi algorithm converges slowly in general, but it has the advantage of being eminently local: each node needs to know only its immediate neighbors' values in order to proceed.

\subsection{Embedded trees algorithm}

In the embedded trees algorithm, the matrix $J:=J_{T}$ corresponds to a spanning tree $\mathcal{G}_{T}$ embedded in the loopy graph $\mathcal{G}[8,9]$. For example, Fig. 1(b) illustrates a minimum-spanning tree for the sensor network in (a). With embedded trees, the inversion of $J_{T}$ can be performed exactly using a message passing algorithm such as belief propagation. Conditions for the convergence of the algorithm are provided in [9]. Iteration over multiple different trees is also possible, and in certain case significantly improves the rate of convergence [9]. The method can further be extended to exactly compute the variance of $\widehat{x}$. Unlike the Jacobi method, the embedded trees algorithm is global; in the Solve step, messages must be passed throughout the entire network in order to compute the next value of $\widehat{x}_{s}^{m}$ at node $s$. 


\section{EMBEDDED TRIANGLES ALGORITHM}

We now present an algorithm that combines the best of the Jacobi and embedded tree methods in the sense that it is both local and fast.

\subsection{Embedded triangles}

When a graphical model contains many loops, it becomes efficient to place in $J$ the elements of $V$ corresponding to small, fully connected subsets of nodes (cliques) of $\mathcal{G}$. In particular, for a triangulated graph, we propose to place in $J$ a set of independent embedded triangles. ${ }^{1}$ (Two triangles are independent if they have no common vertices; see Fig. 1(c) for an example). Let $\tau:=\left\{\tau_{i}\right\}_{i=1}^{M}$ be a set of independent triangles of a loopy graph $\mathcal{G}$, and let $A_{\tau_{i}}$ denote the $3 \times 3$ submatrix of $V$ corresponding to embedded triangle $\tau_{i}$. Assuming a labeling for the nodes, $J$ becomes the block diagonal matrix

$$
J=\left[\begin{array}{ccc}
A_{\tau_{1}} & 0 & 0 \\
0 & \ddots & 0 \\
0 & 0 & D
\end{array}\right],
$$

where $D$ contains the diagonal elements of $V$ corresponding to the residual nodes not included in an embedded triangle. The larger the number $M$ of independent triangles, the faster the convergence.

\subsection{Parallel block-Jacobi algorithm}

With $J$ defined as in (5), the recursion (3)-(4) corresponds to a block-Jacobi iteration. To maximize parallelism, we propose a modified parallel block-Jacobi iteration. Iteration $m$ proceeds as:

Triangles Update: Nodes in independent embedded triangles $s \in \tau$ compute the update $\widehat{y}_{s}^{m}$ as in (3).

Triangles Solve: Each independent embedded triangle $\tau_{i} \in \tau$ solves for $\widehat{x}_{\tau_{i}}^{m}=A_{\tau_{i}}^{-1} \widehat{y}_{\tau_{i}}^{m}$ in parallel.

Residual Update: The residual nodes $t \notin \tau$ compute the update $\widehat{y}_{t}^{m}$ using the latest estimates $\widehat{x}_{s}^{m}, s \in \tau$.

Residual Solve: The residual nodes $t \notin \tau$ solve $\widehat{x}^{m}=\widehat{y}_{t}^{n} / V_{t t}$.

The inversion of each $3 \times 3$ matrix $A_{\tau_{i}}, \tau_{i} \in \tau$ in the Triangles Solve step is performed exactly. During initialization, a cluster-head node $h$ is designated for each triangle $\tau_{i}$. This node gathers the information about $A_{\tau_{i}}$ from its two neighbors and computes and stores the inverse $A_{r_{i}}^{-1}$. During the iterations, $h$ collects the current values of its two neighbors, calculates $A_{\tau_{i}}^{-1} \widehat{y}_{\tau_{i}}^{m-1}$ and sends the updated estimates back to its neighbors.

\subsection{Convergence analysis}

Our specification of the Gaussian HMM model in Section 2.3 ensures that all of the matrix splitting methods described above converge to the true posterior mean $\widehat{x}$. Indeed, if the matrix $V$ is symmetric with negative off-diagonal elements, then the elements of $V^{-1}$ are all positive if and only if $V$ is positive definite [11]. For such a matrix $V$, if $J$ is obtained by setting certain off-diagonals entries of $V$ to zero, then the splitting $V=J-K$ is such that $\rho\left(J^{-1} K\right)<1$ (see Theorem 3.14 in [11]).

\section{SIMULATIONS}

In this section, we compare the performance of the Jacobi, embedded trees, and embedded triangles algorithms using a simulation with the 250-node graphical model in Figure 1(a).

\footnotetext{
${ }^{\mathrm{I}}$ There is nothing sacred about triangles, of course. Graphs containing loops of $L>3$ nodes can be dealt with in exactly the same fashion in an extended embedded polygons algorithm
}

\subsection{Data generation}

We assume the 250 sensor nodes are randomly distributed in the square $[0,1] \times\{0,1]$. We generate the observations $y=x+\epsilon$ with $\epsilon \sim \mathcal{N}\left(0, \sigma^{2} I\right)$, where the variance $\sigma^{2}=4$ and $I$ is the identity matrix. To generate the hidden variables $x$, we first set up the partial correlation coefficients as $r_{s, t \mid \mathcal{V} \backslash\{, t\}\}}=c(1-\operatorname{dist}(s, t))$, with $\operatorname{dist}(s, t)$ the Euclidean distance between nodes $s$ and $t$ and $c$ the largest constant for which the precision matrix $P$ is still positive definite. For the diagonal entries of $P$ we use random variables uniformly distributed between 1 and 2 . This permits us to compute the values $P_{s, t} ; s \neq t$ as described in Section 2.3 and to generate $x \sim N\left(0, P^{-1}\right)$. This specification of $P$ ensures that the elements of $\Sigma=P^{-1}$ are positive (see Section 4.3).

\subsection{Comparison of the three matrix splitting algorithms}

In the embedded trees algorithm, we set $\mathcal{G}_{T}$ to be the Euclidean minimum spanning tree of $\mathcal{G}$; see Figure 1(b). This tree contains the most highly comrelated nodes and hence constitutes a good choice [9]. Since routing in sensor networks can make the discovery of multiple trees difficult and costly, we did not alternate between different trees in the ET algorithm. For the embedded triangles algorithm, we naively clustered the nodes into independent triangles; see Figure 1(c).

We analyzed the convergence of the three algorithms by measuring the residual normalized mean-square error $e^{m}:=$ $\left\|\bar{y}-V \widehat{x}^{m}\right\| /\|\bar{y}\|$, with $\bar{y}=y / \sigma^{2}, V=\Sigma^{-1}+\sigma^{-2} l$, and $m$ the iteration number. We averaged the residual error $e^{m}$ over 50 different realizations of the noise $\epsilon$.

Figures 2(a) and (b) illustrate the superior performance of the embedded triangles algorithm. Figure 2(a) asserts that the residual error for embedded triangles decays faster with increasing number iterations than for the Jacobi and embedded trees. Figure 2(b) reveals that embedded triangles achieves a given residual error using significantly lower inter-node communication energy than either Jacobi or embedded trees. For this comparison, we assume that the energy required by a sensor node $s$ to convey one floating point number to its neighbor $t$ is proportional to $(\operatorname{dist}(s, t))^{2}[2]$. Figure 2(b) indicates that the communication energy-efficient embedded triangles algorithm is well-suited for battery-powered sensor network applications. Indeed, it has been projected that the energy consumed by communications will soon dominate over the energy consumed by sensing and on-board computation $[2,12]$.

\section{HALLMARKS OF EMBEDDED TRIANGLES}

We now summarize some of the desirable features of the embedded triangles algorithm.

Scalable: Since the embedded triangles algorithm operates in parallel over all independent triangles, the amount of time spent on each iteration does not change as we increase the number of nodes $N$ in the network. In contrast, since the embedded trees algorithm relies on sequential message passing along the branches of a tree, its time-per-iteration will increase with the diameter of the network, ${ }^{2}$ which grows with $N[13]$.

Fault tolerant: Embedded triangles can quickly and easily recover from node failures. In the case a node failure, only the two nodes that form an embedded triangle with the failed node need to adapt; these nodes can either seek a new neighboring node not included in another triangle or continue to participate in the iterations as individual nodes (as in Jacobi iterations). All other tri-

\footnotetext{
${ }^{2}$ The diameter of a graph is the length of the longest shortest path between two nodes.
} 


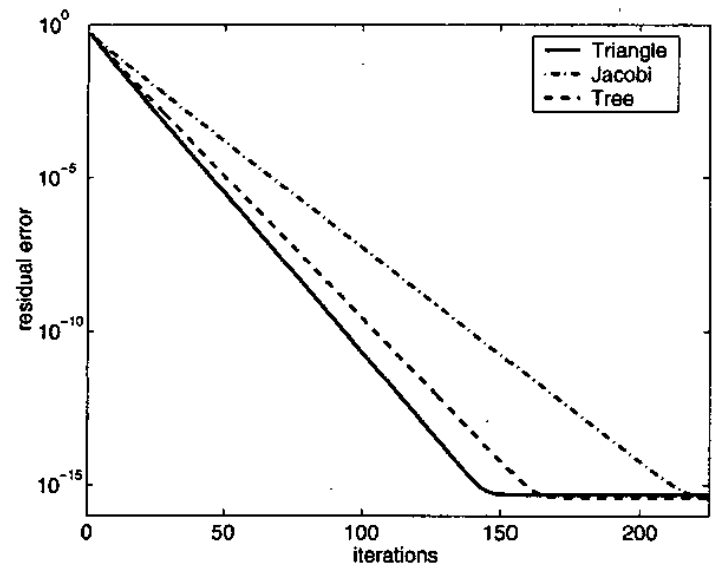

(a) Residual error versus number of iterations



(b) Residual error versus communication energy

Fig. 2. Simulation results for the sensor network in Fig. 1(a)

angles can remain oblivious to the failure and continue with their iterations. In contrast, embedded trees are more fragile, and it will be necessary to employ a routing algorithm to establish a new tree before continuing the iteration.

This same property will enable sensor networks to efficiently self-organize into embedded triangles and begin iterating without expensive up-front route finding.

Energy efficient: As illustrated in Fig. 2(b), the embedded triangles algorithm is communication-energy efficient when compared to the other two matrix splitting algorithms.

\section{DISCUSSION AND CONCLUSIONS}

We have proposed a novel distributed estimation algorithm for loopy Gaussian hidden Markov graphical models. Our iterative approach decomposes a graphical model into independent embedded triangles, performs LMMSE estimation on each triangle, and then updates this estimate by collaborating with neighboring nodes and triangles. Our approach can be interpreted as an extension of the block-Jacobi approach to matrix inversion. For simple spatial correlation models like the one described here, our algorithm enjoys guaranteed convergence.

Our initial results have opened up an array of interesting research directions. Embedded triangles can be trivially generalized by considering $M$-node clusters instead of triangles; we are currently investigating the tradeoffs involved in the choice of the cluster size. Based on the results, we could adapt the cluster size spatially to minimize quantities like inter-node communication energy, iterations required for convergence, etc. Other future avenues include efficiently estimating the variance of our conditional mean estimate, extending the algorithm to track changes over time, optimizing the interaction between distributed estimation schemes and ad hoc wireless network routing protocols, and investigating possible applications to image processing (Markov random fields) and grid computing.

\section{REFERENCES}

[1] D. Estrin, R. Govindan, J. Heidemann, and S. Kumar, "Next century challenges: Scalable coordination in sensor net- works," in Proc. ACM/IEEE MobiCom'99, Aug. 1999, pp. 263-270.

(2) A. Wang and A. Chandrakasan, "Energy-efficient DSPs for wireless sensor networks," IEEE Signal Processing Mag., pp. 68-78, July 2002.

[3] N. A. C. Cressie, Statistics for Spatial Data, Wiley, 1993.

[4] S. L. Lauritzen, Graphical Models, Oxford University press, 1996.

[5] Y. Weiss and W. T. Freeman, "Correctness of belief propagation in Gaussian graphical models of arbitrary topology," Neural Computation, vol. 13, pp. 2173-2200, 2001.

[6] K. Plarre and P. R. Kumar, "Extended message passing algorithm for inference in loopy Gaussian graphical models," $A d$ Hoc Networks, 2002, To appear.

[7] J. Pearl, Probabilistic Reasoning in Intelligent Systems: Networks of Plausible Inference, Morgan Kaufmann, 1988.

[8] M. J. Wainwright, Stochastic Processes on Graphs with Cycles: Geometric and Variational Approaches, Ph.D. thesis, Dept. of Electrical Engineering and Computer Science, MIT, Jan. 2002.

19] E. Sudderth, M. J. Wainwright, and A. S. Willsky, "Embedded trees: Estimation of Gaussian processes on graphs with cycles," Tech. Rep., MIT, 2003.

[10] C. B. Barber, D. P. Dobkin, and H. T. Huhdanpaa, "The quickhull algorithm for convex hulls," ACM Trans. Math. Software, vol. 22, no. 4, pp. 469-483, 1996.

[11] R. S. Varga, Matrix Iterative Analysis, Prentice Hall, 1962.

[12] Y. Xu, S. Bien, Y. Mori, J. Heidemann, and D. Estrin, "Topology control protocols to conserve energy in wireless ad hoc networks," Tech. Rep. 6, University of California, Los Angeles, Center for Embedded Networked Computing, Jan. 2003.

[13] A. Abdalla, N. Deo, and P. Gupta, "Random-tree diameter and diameter-constrained MST," Congressus Numerantium, vol. 144, pp. 161-182, 2000. 